\title{
Anti-arthritic effect of radix Paeoniae rubra herb-acupuncture: a behavioral evaluation on adjuvant-induced arthritis rats
}

\author{
Seung-Tae Kim ${ }^{1}$, Dae-Hyun Hahm ${ }^{1, *}$, Gun-Ho Kim ${ }^{1}$, Jong-Bae Park ${ }^{1}$, Dong-Oh Han ${ }^{1}$, Hi-Joon Park ${ }^{1}$, \\ Insop Shim ${ }^{2}$, Hee-Myung Park ${ }^{3}$, Ki-Hyun Kim ${ }^{4}$, Jang-Hyun Kim ${ }^{4}$, Seong-Hun Choi ${ }^{4}$ and Hye-Jung Lee ${ }^{1}$ \\ ${ }^{1}$ Acupuncture \& Meridian Science Research Center, Kyung-Hee University, Seoul, 130-701; ${ }^{2}$ Department of \\ Integrative Medicine, College of Medicine, The Catholic University of Korea, 505 Banpo-dong, Seocho-gu, Seoul, \\ 137-701; ${ }^{3}$ Department of Veterinary Internal Medicine, College of Veterinary Medicine, Konkuk University, 1 Hwayang- \\ dong, Kwanggin-gu, Seoul, 143-701; ${ }^{4}$ College of Oriental Medicine, Dongkuk University, Kyeongsangbuk-do, \\ 780-714, Korea
}

\section{SUMMARY}

Radix Paeoniae rubra (PR) has been widely used for recovering acute inflammation with swelling and pain in Far East Asian countries. In order to investigate the anti-arthritic effect of PR herbacupuncture, several behavioral parameters such as body weight, knee circumference, squeaking threshold, and weight distribution ratio were investigated in an adjuvant-induced mono-arthritis rat model. We performed PR herb-acupuncture on Zusanli (ST36) of the right hind-leg once every second day for a total of 6 times from the second day of initiating arthritis with adjuvant injection, while the control group was treated with saline in the same way. In the experimental group, body weight significantly increased, knee circumference and squeaking threshold significantly decreased indicating the reduction of edema, and both the pain and inflammatory swelling of the hind paws measured by incapacitance meter decreased. Conclusively, PR herb-acupuncture has more promising effect in alleviating arthritic pain and inflammation than saline-acupuncture in rat model, hence further clinical study is required.

Key words: Radix Paeoniae rubra; Herb-acupuncture; Acupoint; Monoarthritis; Rat

\section{INTRODUCTION}

Arthritis is a common response of joint tissues which have undergone multiform injuries. Its causes include joint deformation, genetic inheritance, physical force, inner-cellular changes and biochemical factors. (Braunwald et al., 2002) Its pathological symptoms usually result from the failure of the cartilage repairing function to keep up with cartilage

*Correspondence: Dae-Hyun Hahm, Acupuncture \& Meridian Science Research Center, Kyung-Hee University, 1 Hoigi-dong, Dongdaemoon-gu, Seoul, 130-701, Korea. Tel: +82312012176; Fax: +82312022175; E-mail: dhhahm@khu.ac.kr breakdown followed by accompanying inflammation. Common symptoms of arthritis are pain, stiffness, limited motion of joints, swelling, and creaking of joints, and the knee joint is the most frequently affected by osteoarthritis (Bennell and Hinman, 2005). Knee osteoarthritis is found in approximately $10 \%$ of the population over the age of 65 (Krohn, 2005). Surgical approaches, several pharmacologic and non-pharmacologic methods are available, and interestingly, non-pharmacologic methods including exercise, weight loss, rest, use of canes and crutches, physical therapy, osteopathic manipulative treatment, and braces received attention. (Rubin, 2005) Besides these, acupuncture and herb- 
acupuncture have been advocated for its clinical effectiveness.

Several types of acupuncture for arthritis have already been studied to some extent: acupuncture (Arichi et al., 1983; Thomas et al., 1991; Laing et al., 2002; Um et al., 2005), electro-acupuncture (Shafshak, 1995), bee venom acupuncture (Kwon et al., 2001; Lee et al., 2004; Vas et al., 2004) and herb-acupuncture (Yeom et al., 2003, 2006). Among the methods, herb-acupuncture has been used for curing various diseases such as osteoarthritis, shoulder pain, and lumbago in Korea. The method is an injection of distilled preparation of herb on acupuncture point (Yeom et al., 2003, 2006). Meanwhile, Zusanli (ST36) acupoint was used in most of those studies, and is known for spasmolytic and analgesic effects on knee joints (Camp, 2001; Kwon et al., 2001; Tillu et al., 2001; Baek et al., 2006; Yeom et al., 2006) and gastrointestinal tract (Lee et al., 2001), and homeostatic effect in the endocrine system and metabolism (Lian et al., 2000; Jang et al., 2003; Choi et al., 2004; Li et al., 2005; Tseng et al., 2005).

We were encouraged by our previous studies of herb-acupuncture and some other studies of Paeoniae rubra (PR), red peony root, which has been used to alleviate a febrile disease, to manifest blood stagnation, and to recover acute inflammation with red swelling and pain from external injury (Bensky and Gamble, 1993; Ahn, 1998; Korean Institute of Herbal Acupuncture, 2000). In this study, the antiarthritic effects of PR herb-acupuncture were investigated in an adjuvant-induced mono-arthritis rat. To assess the arthritic symptoms of pain and inflammation, several preclinical and behavioral tests, such as body weight, knee circumference, and squeaking threshold, weight distribution ratio, were measured and their accuracy and effectiveness as an arthritic indicator were compared.

\section{MATERIALS AND METHODS}

\section{Animals}

Female Sprague-Dawley rats (Samtaco Co., Osan,
Korea), weighing 120-140 g, were used for the experiments. Animals were given a period of 1 week to adjust to the new environment $\left(22 \pm 2^{\circ} \mathrm{C}\right.$, $45-60 \%$ humidity, and $12 \mathrm{~h}$ light/ $12 \mathrm{~h}$ darkness cycle, raising 5 - 6 animals per cages) and provided with hard food (Oriental Co., Korea) and water ad libitum. The experimental procedures were carried out according to the animal care guidelines of the $\mathrm{NIH}$ and the Kyung Hee University Institutional Animal Care and Use Committee.

\section{Arthritis induction}

In this study, a mono-arthritis inducing protocol, that is, a fixed quantity of the antigen was injected into the synovial cavity of the knee joint, was used (Neto et al., 1999; Yu et al., 2002; Bäckdahl et al., 2003). After anesthetized with halothane, the rats were injected with $80 \mathrm{mg}$ of arthritis-inducing adjuvant with a disposable 1 ml-syringe (26 guage needle, Korea vaccine Co., Korea) at the synovial cavity of the right knee joint. The arthritis-inducing adjuvant was 1:1 mixture of saline and heat-killed Mycobacterium tuberculosis H37 RA (Difco Co., Detroit, MI, USA), emulsified in $0.15 \mathrm{ml}$ squalene (SigmaAldrich Chemical Co., MO, USA). An injected region was sterilized by $70 \%$ ethanol.

\section{Herbal component of herb-acupuncture}

The dried herb of Radix Paeoniae rubra (PR) was kindly supplied from Kyung-Hee University Oriental Hospital (Seoul, Korea), and voucher specimens have been deposited in the Herbarium of KyungHee University. The distilled water extracts of PR for herb-acupuncture treatment were manufactured in an ampoule by Korean Institute of Herbal Acupuncture (Seoul, Korea). The manufacturing processes sequentially proceeded by washing the herb by distilled water, boiling, evaporation, condensation, filtering the extract with $0.45 \mu \mathrm{m}$ and $0.1 \mu \mathrm{m}$ filter films, refrigerated precipitation for mineral exclusion using $0.9 \% \mathrm{NaCl}$, $\mathrm{pH}$ regulation by $\mathrm{Na}_{3} \mathrm{PO}_{4}$ and citric acid, and high pressure sterilization steps, as previously reported (Korean 
Institute of Herbal Acupuncture; 2000; Yeom et al., 2003), which were always under the quality controls of no suspending particles using a visible spectrophotometer. The extraction was started with $200 \mathrm{~g}$ of dried PS and finally produced $300 \mathrm{ml}$ of PS distillates for herb-acupuncture. By the regulation of Korean Institute of Herbal Acupuncture, the PS distillates were passed the germ-free, antihistamine, and endotoxin-free tests, and thus accepted as an s.c. injection grade for human.

\section{Experimental groups}

Experimental animals were divided into three groups:

1) no-treated, non-arthritic animals (NOR, $n=10)$;

2) PR-treated/ST36 acupoint (Zusanli) arthritic animals (PR, $\mathrm{n}=10)$; and 3) saline-treated/ST36 acupoint (Zusanli) arthritic animals (SAL, $\mathrm{n}=10$ ). After induced, the adjuvant-induced rats were randomly divided into two groups (PR and SAL) on day 2. Degrees of redness and swelling on the joints were similar between the two groups, and rats having symptoms which were too severe or not induced were excluded.

\section{Herb-acupuncture therapy}

The PR herb-acupuncture treatment initiated on day 2 was the post adjuvant injection and executed to the right leg every second days for 12 consecutive days, for a total of 6 times. The $0.05 \mathrm{ml}$ of the water extract of PR was subcutaneously injected into ST36 acupoint using $0.5 \mathrm{ml}$ insulin syringe ( 30 gauges, BD Biosciences Co., CA, USA). The acupoint is located near the knee joint of the hind limb, $2 \mathrm{~mm}$ lateral to the anterior tubercle of the tibia. The injected region was sterilized by $70 \%$ ethanol just prior to the treatment.

\section{Behavioral \& pre-clinical parameters}

From day 0 to day 14 , body weight was regularly measured every second day by a digital balance, especially on day 1 . After measuring body weight, the circumference of the knee joint was measured twice at the lateral and medial ligament region of the joint, using a flexible and elastic band. Calculating the average of results, we compared the difference between the average circumference of an arthritisinduced knee joint (right side) and that of a healthy joint circumference (left side). Then, squeaking threshold was investigated by slowly extending and flexing the arthritic-induced knee joint of a rat five times. The recorded results were then calculated. Each squeak was counted as one point, and the highest point was 10 . The interval of the extension-flexion test was $5 \mathrm{~s}$. A rat was placed into a specifically created sloping acrylic box, and the lower limbs were positioned onto an IITC 600 incapacitance meter (IITC Life Science Inc, CA, USA). The average of the weight loaded at the each lower limb was measured for $5 \mathrm{~s}$ and repeated four times. Then, the average of the series was utilized. Using this method, weight distribution ratio was measured on day $0,2,8,14$, and was then calculated using the next formula.

$$
\begin{aligned}
& \text { Weight Distribution Ratio } \\
& =\frac{\text { weight borne by ipsilateral paw }}{\text { total weight borne by both paws }} \times 100
\end{aligned}
$$

\section{Data analysis}

The data were presented as the mean \pm S.E.M. The significance of the differences between groups was assessed by a one-way analysis of variance (ANOVA), followed by the Tukey HSD post-hoc analysis. Difference was considered significant at $P<0.05$.

\section{RESULTS}

Using an adjuvant-induced mono-arthritis rat, the therapeutic effect of PR herb-acupuncture for arthritis was investigated in this study. Behavioral tests such as body weight, knee circumference, and squeaking threshold were measured, and those results were compared with weight distribution ratio. 


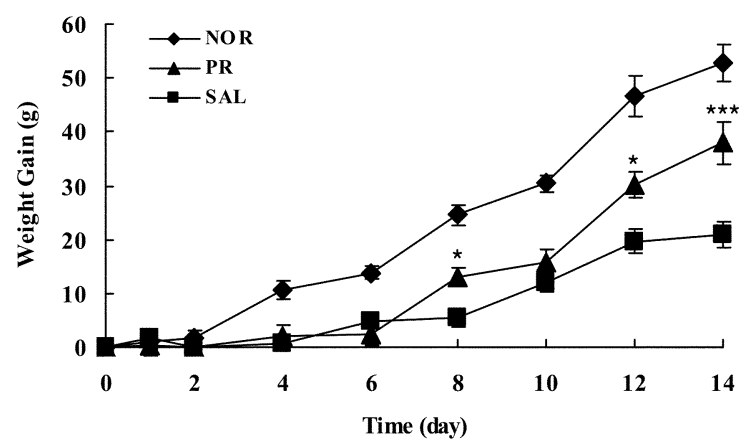

Fig. 1. Effect of radix PR herb-acupuncture therapy on body weight of adjuvant-induced mono-arthritic rat. The NOR, PR and SAL mean the experimental groups treated with no arthritis induction $(n=5)$, the PR-acupuncture at Zusanli (ST36) point $(n=10)$, and saline herb-acupuncture at the same acupoint $(n=10)$, respectively. The $\mathrm{Y}$-axis was indicated by gain of body weight after day 0 . The mean numbers of body weights were calculated and analyzed by repeated ANOVA followed by the Tukey HSD post hoc test for further confirmation. Significance was indicated as ${ }^{*} P<0.05$ vs. SAL group and ${ }^{* * *} P<0.01$ vs. SAL group.

\section{Body weight}

Fig. 1 displays the effect of PR herb-acupuncture therapy on the body weight of an adjuvant-induced mono-arthritis rat. Until day 2, there was little increase in body weights of all rats involved in the experiment. From day 2, the body weight of the PR group continually increased, and during days 6 and 14, a significant increase in weight was observed in the PR group as compared to that of SAL group (day 8: $P<0.05$, day 14: $P<0.01$ ). In terms of water and food consumption, there were no significant differences amongst the three groups (data not shown). In the case of the NOR group, the weight increase was more pronounced, as compared to those in the arthritis-induced groups (PR and SAL).

\section{Knee circumference}

For measuring the degree of edema in an arthritisinduced knee joint, the variation of knee circumference was analyzed. Fig. 2 displays the effect of PR herbacupuncture therapy on the knee circumference of a adjuvant-induced mono-arthritic rat. A variation in the knee circumference of the NOR group was

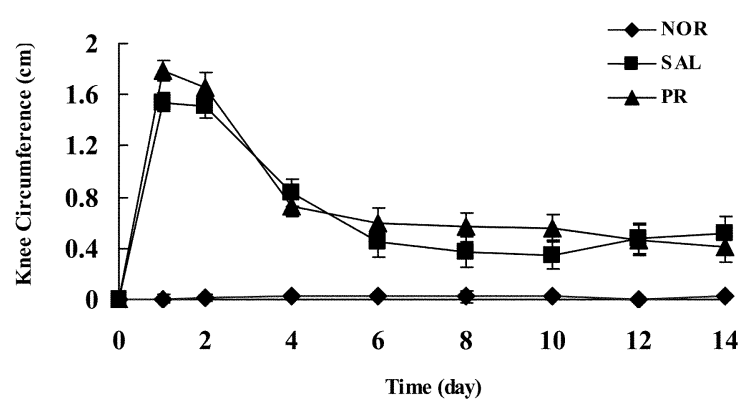

Fig. 2. Effect of radix PR herb-acupuncture therapy on knee circumference of adjuvant-induced mono-arthritic rat. The NOR, PR and SAL mean the experimental groups treated with no arthritis induction $(n=5)$, the PR-acupuncture at Zusanli (ST36) point $(\mathrm{n}=10)$, and saline herb-acupuncture at the same acupoint $(\mathrm{n}=$ $10)$, respectively. The Y-axis was indicated by gain of knee circumference after day 0 . The mean numbers of knee circumference data were calculated and analyzed by repeated ANOVA followed by the Tukey HSD post hoc test for further confirmation.

not observed during the experimental period (14 days). In the PR and SAL groups, a decrease was noticed beginning from day 2 , and the circumference decreased less than $0.2 \mathrm{~cm}$ after day 6 . However, a significant decrease between the two groups was not observed during the first 10 days.

\section{Squeaking threshold}

In the case of the NOR group, from 0 day to 14 day (conclusion of the experiment), most of the squeaking scores were zero. However, a difference of squeaking scores between the PR and SAL groups could be observed. The score of the SAL group was almost 10, and it decreased a little after day 12. On the other hand, the score of the PR group maintained a score of about 7 after day 6 , which was significantly less than that of the of SAL group $(P<0.001)$. (Fig. 3$)$

\section{Weight distribution ratio}

Fig. 4 shows the effect of PR herb-acupuncture therapy on weight distribution between the paws of an adjuvant-induced mono-arthritic rat. The weight distribution ratios of the unilateral paws in the NOR group were nearly $50 \%$. In case of the PR 


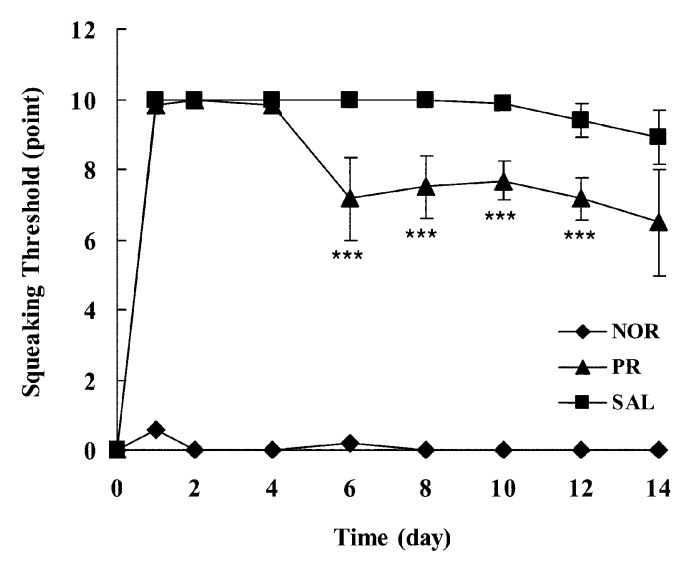

Fig. 3. Effect of radix PR herb-acupuncture therapy on squeaking behavior of adjuvant-induced mono-arthritic rat. The NOR, PR and SAL mean the experimental groups treated with no arthritis induction $(\mathrm{n}=5)$, the PR-acupuncture at Zusanli (ST36) point $(n=10)$, and saline herb-acupuncture at the same acupoint $(\mathrm{n}=$ 10), respectively. The mean numbers of squeaking data were calculated and analyzed by repeated ANOVA followed by the Tukey HSD post hoc test for further confirmation. Significance was indicated as ${ }^{* * *} P<0.001$ vs SAL group.

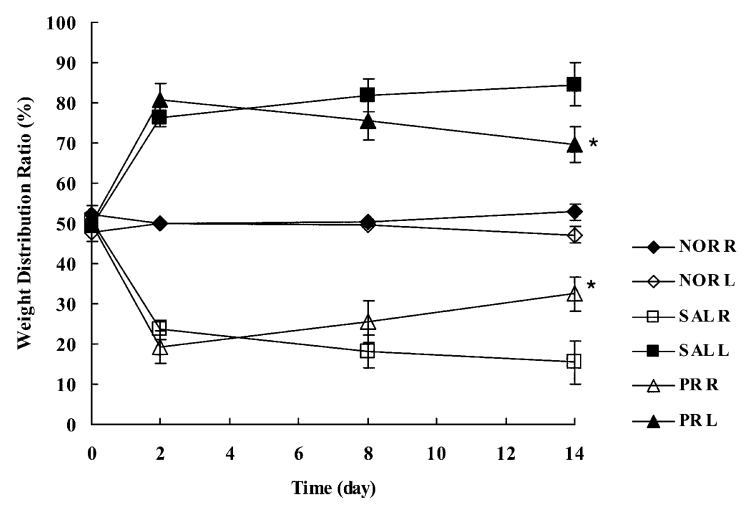

Fig. 4. Effect of radix PR herb-acupuncture therapy on weight distribution of adjuvant-induced mono-arthritic rat. ' $L$ ' mean the left side leg, and ' $R$ ' mean the right side leg. The NOR, PR and SAL mean the experimental groups treated with no arthritis induction $(n=5)$, the PR-acupuncture at Zusanli (ST36) point $(n=10)$, and saline herb-acupuncture at the same acupoint $(\mathrm{n}=$ 10), respectively. The weight distribution ratios of the unilateral paws in NOR group were nearly $50 \%$. The PR or saline treatment was started on day 2 and executed every second day for 12 days. The mean numbers of weight distribution data were calculated and analyzed by repeated ANOVA followed by the Tukey HSD post hoc test for further confirmation. Significance was indicated as ${ }^{*} P<0.05$ vs SAL group. group, the weight distribution ratio of the arthritisinduced paw (right side) in the early stage was $20 \%$. However, a change in the ratio could be seen from day 8 , and the change was significant $(30 \%$, $P<0.05$ vs SAL group) on day 14 . In comparison, the ratio of an arthritis-induced paw in the SAL group was maintained at about $30 \%$ in the early stage, however, the ratio continued to decrease during the whole experimental period, and it was at about $15 \%$ on day 14 . This means that the saline injection into the acupoint did not produce a medicinal effect and the arthritic symptoms worsened as time passed.

\section{DISCUSSION}

Herb-acupuncture therapy is one of the newer therapies, is it widely used in Korean traditional medicine. It has adopted both the medical benefits of acupuncture and herbal therapy (Kwon et al., 2001; Yeom et al., 2003; Lee et al., 2004; Yeom et al., 2006). In this study, radix PR was selected as the material for the herbal extract. It contained paeoniflorin, albiflorin, paeonn, paeonol, benzoic acid, tannin, etc. This extract has been widely used in traditional Korean and Chinese medicines for thousands of years to invigorate the blood and remove blood stasis, to clear heat and cool the blood, and to clear liver fire (Bensky and Gamble, 1993; Ahn, 1998). Concretely, it has been known to be an effective medicine in alleviating febrile disease (Genfa et al., 2005), manifesting blood stagnation such as dysmenorrhea and amenorrhea (Xie et al., 2005), dispelling abdominal pain and immobile abdominal masses, reducing skin blotches, stopping spasms, and in recovering acute inflammation with red swelling and severe pain (Ahn, 1998; Bensky and Gamble, 1993; Korean Institute of Herbal Acupuncture, 2000). Therefore, it was suitable choice as a medicinal herb for arthritis treatment.

In this study, the effect of PR herb-acupuncture therapy was investigated by using an adjuvantinduced mono-arthritic rat. The parameters such as 
body weight, knee circumference, and squeaking score were measured, and the results were compared to those of the weight distribution ratio. To assess behavioral and pre-clinical arthritic symptoms of a pain animal model, the following experimental methods are generally used - body weight measuring, acetone atomizing and hot plate test, heat sensitivity measuring method of the paw, and the Von Frey hair test. For measuring the degree of edema in the inflamed region of the body, mainly the paw volume, knee circumference, squeaking threshold, and articular index are analyzed. However, just as various analgesia equipment have their merits and demerits, the experimental limits, such as over subjective inclination, measuring the degree of pain and edema, requirement of general anesthesia can also have a similar effect. Therefore more than two methods were generally used at the same time, in order to improve experimental accuracy. For overcoming certain limits, an incapacitance meter, a new type of analgesia meter which is used to measure the weight difference between both soles of the arthritic rat's feet, was used for exclusion of subjective inclination, analyzing pain and edema at once, and the use of anesthesia was not necessary (Schott et al., 1994; Hay et al., 1997).

In the case of body weight, the weights of all groups were not increased during first 2 days. After that, the weights were begun to increase gradually, and a significant increase was observed compared to the SAL group on day 6 and 14. The animal model in this study had a short arthritisinduced and recovery period, compared to the chronic poly-arthritic animal model. In the results, the experimental schedules had to be designed within a 2 week span, in order to investigate the therapeutic effects. Also, it was difficult to observe the loss of body weight due to the local injection of adjuvant for inducing a mono-arthritis, and to observe the recovery of body weight by medical therapy in a mono-arthritic rat. For these reasons, the efficacy of the PR herb-acupuncture therapy in terms of body weight recovery was not significantly verified in this study.

For investigating the degree of edema caused by local induction of arthritic inflammation, the variation of knee circumference was measured. Between the PR and SAL groups, the decrease in the knee circumferential variation was observed on day 2 , and the variation decreased lesser than 0.2 $\mathrm{cm}$ from day 6 . After day 12 , compared to the SAL group, the PR group had decreased more, but not so much that it was statistically significant. Kwon et al. (2001) was reported that edema was dramatically decreased after injecting bee venom into ST36, and Yeom et al. (2006) was also reported that inflammation decreased after injecting water extracts of Hominis placenta using a poly-arthritic animal model. In this study, a significant decrease of knee circumference by the PR herb-acupuncture was not observed, but the tendency to decrease was displayed. These results indicated that PR herb-acupuncture was somewhat effective for adjuvant-induced mono-arthritis, although a significant decrease was not observed. In order to obtain a more solid verification, longer-term experiments must be conducted studied.

Squeaking threshold scoring, using modified methods of Park (2001) and Yu (2002), were used for analyzing rigidity and pain of knee arthritis. In a knee joint, arthritis was the cause of sensitized pain receptors, induced rigidity, and restricted extension-flection. Consequently, squeaking was not induced in normal animals by extension-flection of knee joint, but was induced in arthritis-induced animals. In this study, after day 6 , squeaking scores in the PR group were significantly different from those in the SAL group. This would indicate that PR herb-acupuncture therapy was effective for alleviating rigidity and pain as an arthritic symptom. However, a significant decrease was not observed in the knee circumference test. These results also indicated that the PR herb-acupuncture therapy was more effective for alleviating arthritic pain than for reducing inflammation and arthritic edema.

In the end, using an incapacitance meter, a paw 
pain measuring unit, the efficacy of the PR herbacupuncture therapy for treating arthritis was investigated. This device was designed to detect the difference of weight distribution between the two paws of an arthritis-induced rat, referring to Hay (1997) and Schott (1994). In this animal model, the pain only occurred at the arthritis-induced paw (right side) when body weight was placed onto both hind paws. To avoid or minimize the pain, the rats shifted their body weight onto the healthy paw (left side). Therefore, the difference of weight distribution between the two paws was attributed to an action for avoiding pain. In the results, the wider the difference in weight distribution ratio means the more painful a paw had become, due to the arthritis.

Before the arthritis induction, the weight distribution between two paws was equal, that is 5:5. After arthritis induction, the ratio rapidly changed to $8: 2$ on day 2. In the PR group, the efficacy of the PR herb-acupuncture therapy was observed on day 8 , and the ratio improved to 7:3 on day 14 . After that, the ratio nearly approached 5:5 again. In the case of the SAL group, during the experimental period (14 days), the ratio was maintained at about 8:2. It suggested that a spontaneous cure effect had not appeared in this acute-arthritis rat model during the 14 days, and that the PR herb-acupuncture therapy had been effective for pain in the arthritisinduced knee joint, at least in terms of reducing pain.

In spite of a few demerits, such as requiring an adaptation period for maintaining the standing posture of a rat, there were many advantages of this device. First of all, anesthesia is not necessary, so the arthritic symptoms, such as pain, edema, and rigidity, can be synthetically expressed. It was therefore suggested that the analyzing values of this device were sufficient as a pain measuring method.

In conclusion, for investigating the anti-arthritic effects of PR herb-acupuncture, body weight, knee circumference, squeaking threshold, and weight distribution ratio of an adjuvant-induced monoarthritis rat model were measured, as compared to those of that had a saline injection administered to the same acupoint as a control. In regards to the arthritic model development, the adjuvant injection to the knee joint produced arthritic symptoms such as edema, pain, stiffness and rigidity. The PR herbacupuncture therapy continually increased the body weight, and a significant increase of the body weight was observed in the PR group, when compared to SAL group. The therapy began to decrease the variation of knee circumference from day 2 , but a significant decrease was not observed. In an extension-flection test, a type of pain analysis method for indicating the degree of arthritic severity, the squeaking score had decreased significantly. And the efficacy of the PR herb-acupuncture was synthetically verified in pain control by an incapacitance meter, a new type of paw pain measuring device analyzing the difference in weight distribution between two paws.

Taken together, the PR herb-acupuncture therapy was more effective for the alleviation of pain and inflammation than the simple acupuncture such as saline herb-acupuncture as previously reported (Kwon et al., 2001; Kang et al., 2002; Lee et al., 2004). And the injection of highly purified herbal distillates into the specific acupoint is essential to achieve herb-acupuncture therapy.

\section{ACKNOWLEDGEMENTS}

This work was supported by the SRC program of KOSEF (R11-2005-014).

\section{REFERENCES}

Ahn DK. (1998) Illustrated Book of Korean Medical Herbs, p. 665, Kyo-Hak publishing, Seoul.

Arichi S, Arichi H, Toda S. (1983) Acupuncture and Rehabilitation (III) Effects of Acupuncture Applied to the Normal Side on Osteoarthritis Deformans and Rheumatoid Arthritis of the Knee and on Disorders in Motility of the Knee Joint after 
Cerebral Hemorrhage and Thrombosis. Am. J. Chin. Med. 11, 146-149.

Bäckdahl L, Ribbhammar U, Lorentzen JC. (2003) Mapping and functrional characterization of rat chromosome 4 regions that regulate arthritis models and phenotypes in congenic strains. Arthritis Rheum. 48, 551-559.

Baek YH, Huh JE, Lee JD, Choi DY, Park DS. (2006) Antinociceptive effect and the mechanism of bee venom acupuncture (Apipuncture) on inflammatory pain in the rat model of collagen-induced arthritis: Mediation by alpha2-Adrenoceptors. Brain Res. 16, 1073-1074.

Bennell K, Hinman R. (2005) Exercise as a treatment for osteoarthritis. Curr. Opin. Rheumatol. 17, 634-640.

Bensky D, Gamble A. (1993) Chinese Herbal Medicine: Materia Medica, pp. 277-278, Eastland press, Vista.

Braunwald E, Fauci AS, Kasper DL, Hauser SL, Longo DL, James JL. (2002) Harrison's manual of medicine, p. 748, McGraw-Hill, New York.

Article in Book: Camp AV. (2001) Acupuncture for rhematological problems. In: Medical Acupuncture, edited by Filshie J, White A, pp. 353-362, KCM press, Seoul.

Choi GS, Han JB, Park JH, Oh SD, Lee GS, Bae HS, Jung SK, Cho YW, Ahn HJ, Min BI. (2004) Effects of Moxibustion to Zusanli (ST36) on Alteration of Natural Killer Cell Activity in Rats. Am. J. Chin. Med. 32, 303-312.

Genfa L, Jiang Z, Hong Z, Yimin Z, Liangxi W, Guo W, Ming H, Donglen J, Lizhao W. (2005) The screening and isolation of an effective anti-endotoxin monomer from radix Paeoniae rubra using affinity biosensor technology. Int. Immunopharmacol. 5, 10071017.

Hay CH, Trevethick MA, Wheeldon A, Bowers JS, de Belleroche JS. (1997) The potential role of spinal cord cyclooxygenase-2 in the development of Freund's complete adjuvant-induced changes in hyperalgesia and allodynia. Neuroscience 78, 843-850.

Jang MH, Shin MC, Lim BV, Kim HB, Kim YP, Kim EH, Kim H, Shin MS, Kim SS, Kim CJ. (2003) Acupuncture Increases Nitric Oxide Synthase Expression in Hippocampus of Streptozotocininduced Diabetic Rats. Am. J. Chin. Med. 31, 305-313.

Kang SS, Park SC, Choi SH. (2002) The Effect of Whole Bee Venom on Arthritis. Am. J. Chin. Med.
30, 73-80

Korean Institute of Herbal Acupuncture. (2000) The guide book of herb acupuncture treatment, pp. 12, 18-33, 157-159, Korean Institute of Herbal Acupuncture, Seoul.

Krohn K. (2005) Footwear alterations and bracing as treatments for knee osteoarthritis. Curr. Opin. Rheumato. 17, 653-656.

Kwon YB, Lee JD, Lee HJ, Han HJ, Mar WC, Beitz AJ, Lee JH. (2001) Bee venom Injection into an Acupuncture Point Reduces Arthritis Associated Edema and Nociceptive Responses. Pain 90, 271-280.

Laing AJ, Mullett H, Gilmore MF. (2002) Acupunctureassociated arthritis in a joint with an orthopaedic implant. J. Infect. 44, 43-44.

Lee CH, Jung HS, Lee TY, Lee SR, Yuk SW, Lee KG, Lee BH. (2001) Studies of the Central Neural Pathways to the Stomach and Zusanli (ST36). Am. J. Chin. Med. 29, 211-220.

Lee JD, Kim SY, Kim TW, Lee SH, Yang HI, Lee DI, Lee YH. (2004) Anti-inflammatory effect of bee venom on type II collagen-induced arthritis. Am. J. Chin. Med. 32, 361-367.

Li H, Tang CZ, Li SH, Zhang Z, Chen SJ, Zhang JW. (2005) Effects of thread embedding therapy on nucleotides and gastrointestinal hormones in the patient of chronic gastritis. Zhongguo Zhen Jiu 25, 301-303.

Lian YL, Chen CY, Hammes M, Kolster BC. (2000) The Seirin Pictorial Atlas of Acupuncture, pp. 74-75, Könemann.

Neto FL, Schadrack J, Ableitner A, Castro-Lopes JM, Bartenstein P, Zieglganxberger W, Tolle TR. (1999) Supraspinal metabolic activity changes in the rat during adjuvant monoarthritis. Neuroscience 94, 607621.

Park HJ. (2001) The effect of electroacupuncture on the chronic monoarthritis in rats. Thesis for the degree of doctor of philosophy in oriental medicine, graduate school of east-west medical science, Kyunghee university, Seoul.

Rubin BR. (2005) Management of osteoarthritic knee pain. J. Am. Osteopath. Assoc. 105, S23-28.

Shafshak TS. (1995) Electroacupuncture and Exercise in Body Weight Reduction and their Application in Rehabilitating Patients with Knee Osteoarthritis. Am. J. Chin. Med. 23, 15-25. 
Schott E, Berge OG, Angeby-Moller K, Hammarstrom G, Dalsgaard CJ, Brodin E. (1994) Weight bearing as an objective measure of arthritic pain in the rat. $J$. Pharmacol. Toxicol. Methods 31, 79-83.

Thomas M, Eriksson SV, Lundeberg T. (1991) A Comparative Study of Diazepam and Acupuncture in Patients with Osteoarthritis Pain: A Placebo Controlled Study. Am. J. Chin. Med. 19, 95-100.

Tillu A, Roberts C, Tillu S. (2001) Unilateral versus bilateral acupuncture on knee function in advanced osteoarthritis of the knee--a prospective randomised trial. Acupunct. Med. 19, 15-18.

Tseng CS, Shen WC, Cheng FC, Chen GW, Li TC, Hsieh CL. (2005) Dynamic change in energy metabolism by electroacupuncture stimulation in rats. Am. J. Chin. Med. 33, 767-778.

Um SW, Kim MS, Lim JH, Kim SY, Seo KM, Nam TC. (2005) Thermographic evaluation for the fficacy of acupuncture on induced chronic arthritis in the dog. J. Vet. Med. Sci. 67, 1283-1284.

Vas J, Perea-Milla E, Mendez C. (2004) Acupuncture and moxibustion as an adjunctive treatment for osteoarthritis of the knee--a large case series. Acupunct. Med. 22, 23-28.

Xie WG, Ma XC, Shao NS. (2005) Preliminary study on change of serum proteome in noxious heat blood stasis syndrome treated by radix Paeoniae rubra. Zhongguo Zhong Xi Yi Jie He Za Zhi 25, 520-524.

Yeom MJ, Lee HC, Kim GH, Lee HJ, Shim I, Oh SK, Kang SK, Hahm DH. (2006) Anti-arthritic effects of Ephedra sinica STAPF herb-acupuncture: inhibition of lipopolysaccharide-induced inflammation and adjuvant-induced polyarthritis. J. Pharmacol. Sci. 100, 41-50.

Yeom MJ, Lee HC, Kim GH, Shim I, Lee HJ, Hahm DH. (2003) Therapeutic effects of Hominis placenta injection into an acupuncture point on the inflammatory responses in subchondral bone region of adjuvant-induced polyarthritic rat. Biol. Pharm. Bull. 26, 1472-1477.

Yu YC, Koo ST, Kim CH, Lyu YS, Grady JJ, Chung JM. (2002) Two variables that can be used as pain indices in experimental animal models of arthritis. J. Neurosci. Methods 115, 107-113. 